\title{
Applications of Wavelet Transform and Wavelet Packet Transform to Detect the Crack in Micro Beams
}

\author{
Ravikumar.K ${ }^{1}$, Ithaya Priya.B ${ }^{2}$ \\ ${ }^{1}$ Engineering Mathematics, Faculty of Engineering and Technology, Annamalai University' \\ Annamalai Nagar, Tamil Nadu,608002. \\ ${ }^{2}$ Department of Mathematics, Annamalai University, Annamalai Nagar, Tamil Nadu,608002. \\ ravik672003@yahoo.com, priyatrp25@gmail.com
}

Article History: Received: 10 November 2020; Revised 12 January 2021 Accepted: 27 January 2021; Published online: 5 April 2021

\begin{abstract}
Micro beams found to be an optimistic device for several applications in various MEMS device like actuators, diaphragms, sensors and accelerometers. Its high sensitivity, accuracy and precision made this to be a basic functional unit of most MEMS devices. Micro beams shows difference in its vibrational frequency, stress and deflection over various input conditions. Occurrence of defects in the miniaturized structure can show major impact in its performance and sensitivity. Hence it is very essential to detect any defects presents in the micro device structure. In this study an eminent method of identifying the presence of cracks and its exact locations by interpreting the change in deflection and stress experienced by the micro beam using wavelet transform was illustrated. Wavelet transform and its packet decomposition is one among the efficient mathematical tool for analyzing signals in both frequency and time domain. The proposed device structure was designed and modeled using COMSOL Multiphysics simulation software. From the obtained results the location of the cracks which appears in micro cantilever structure due to various working conditions can be perfectly identified using wavelet transform analysis.
\end{abstract}

Keywords: Wavelet Analysis, WPT, Crack Detection, Micro Beam, MEMS, COMSOL.

\section{Introduction}

Wavelet transform (WT) is an eminent approach for signal analysis by the scaled and shifted wavelets which is being applied to a wide range of engineering applications. The term wavelets was first introduced in early 1980's by J.Morlet and A.Grossmann, which was very much concentrated over theoretical mathematics than its applications[1]. Later Daubachies and Mallet redefined the wavelets for various applications like time frequency spectral analysis, digital signal processing, data compression and image processing. Till that Fourier analysis was the dominating tool in signal processing. The Fourier transform (FT) disintegrate the function in a range of low to high frequency components, which have a narrow support in one domain[2]. But the wavelet has basis functions on both domains. Fourier signal does not contains any local data and found to be in appropriate in analyzing the time varying signals. An effective signal processing techniques should have potential to analyze continuous and transient signals in both time and frequency domains[3]. But FT can provide efficient signal analysis in frequency domain, but it is inappropriate for time varying signals due to unavailability of local data. WT represents to be an efficient and alternative method to FT. This deficiencies experienced in Fourier analysis made the researches to find the alternative to overcome this issues. WT has the potential to provide accurate time and frequency resolutions of a signal. This instinctive feature of wavelet transform made it very popular for various engineering applications[4]. WT is found to be the direct alternative to FT in time-frequency analysis of discrete signals. Since WT has its origin in mathematical theory, its application and implementation of very simpler as the theory suggests[5].

Wavelet packet transform(WPT) which is the generalization of WT is flexible over time-frequency plane in analyzing signals, found to be more dominant in applications[6]. Wavelet packets which can be generalized using the link between approximations, multi-resolution analysis and wavelets were brought out and explained by coifman et al. WPT can effectively provide the details of original signals in all the levels and in all 
dimensions. WPT provides complete multiple analysis of the input signal based on wavelet functions[7]. The analysis and classification is well performed and reproduced by the limited number of wavelet coefficients for the relevant signal input. WPT is the emerging successive mathematical analysis concept in the field of fault and crack diagnosis because of their superior frequency localization than wavelets.

In this present study, we utilize the wavelet packets to analyze the output signal from a micro beam to locate any damages or cracks present. The wavelet coefficients will reflect with peaks when any changes or discontinuities occur in data[8]. By the use of such ridges and peaks in wavelet analysis, we could exactly locate the cracks and damages present in the micro device. Dynamic characteristics of the device will reflect with respect to the damage or cracks over the surface[9,10]. Here we had compared devices with various cracks present in the device surface. The device with structural damages is compared with healthy device and the presence of location is identified by use of wavelet transform analysis and wavelet packet transform.

\section{Wavelet Packet Transform}

The wavelet packet transform (WPT) is a famous mathematical tool that has special merits over Fourier transform in analyzing non-stationary signals. It provides arbitrary time-frequency resolution which adopts redundant basic functions. WPT is more advanced version of continuous wavelet transform and can make a suitable level by level resolution for each signal. Wavelet packets divide the frequency axis into finer and pixel parts than the DWT. In Wavelet Packet Decomposition the discrete signals is passed through more number of filters than the discrete wavelet transform[11]. In wavelet analysis, the signal is divided into detail and approximation. But in wavelet packet analysis, the detail as well as approximation can be differentiated which offers a superior range of possibilities for signal processing.

\section{Figure.1 Comparison of wavelet decomposition tree(a) and wavelet packet decomposition tree (b) at level}

2.

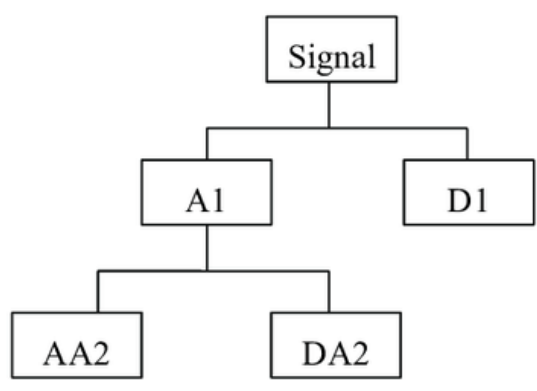

(a)

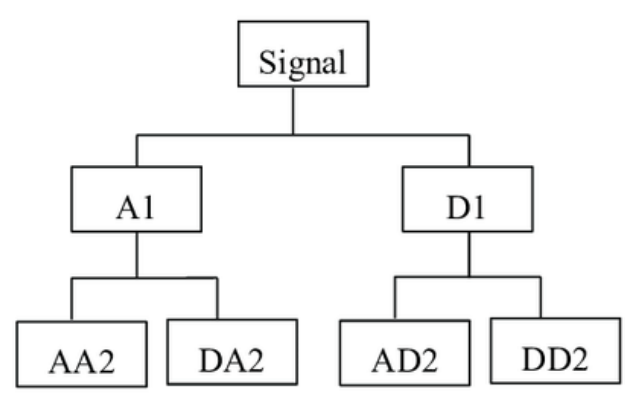

(b)

Wavelet decomposition is made to resolve high frequency components within a small time window, while low frequency component requires large time. Here the low frequency component consumes large time interval whereas a high frequency component needed cycle in a much shorter interval[12]. Therefore, slow varying components can only be identified over long time intervals but fast varying components can be identified over short time intervals. Wavelet decomposition can be treated as a continuous time wavelet decomposition which is sampled at different frequencies at each level or scale. 
Wavelet packet analysis is also considered to be an extension of the DWT and it turns out that the DWT is only one of the much possible decomposition that could be performed on the signal. Instead of just decomposing the low frequency components, it is therefore possible to divide the whole time frequency plane into various time frequency pieces. The advantage of wavelet packet analysis is that it is possible to fuse the different levels of decomposition in order to achieve the optimum time frequency representation [13]. In classical wavelet decomposition the image is break into an approximation and details images[14]. The approximation separates itself into a second level of approximation and details as shown in Figure.2.

\section{Figure.2 2D Wavelet Packet Decomposition}

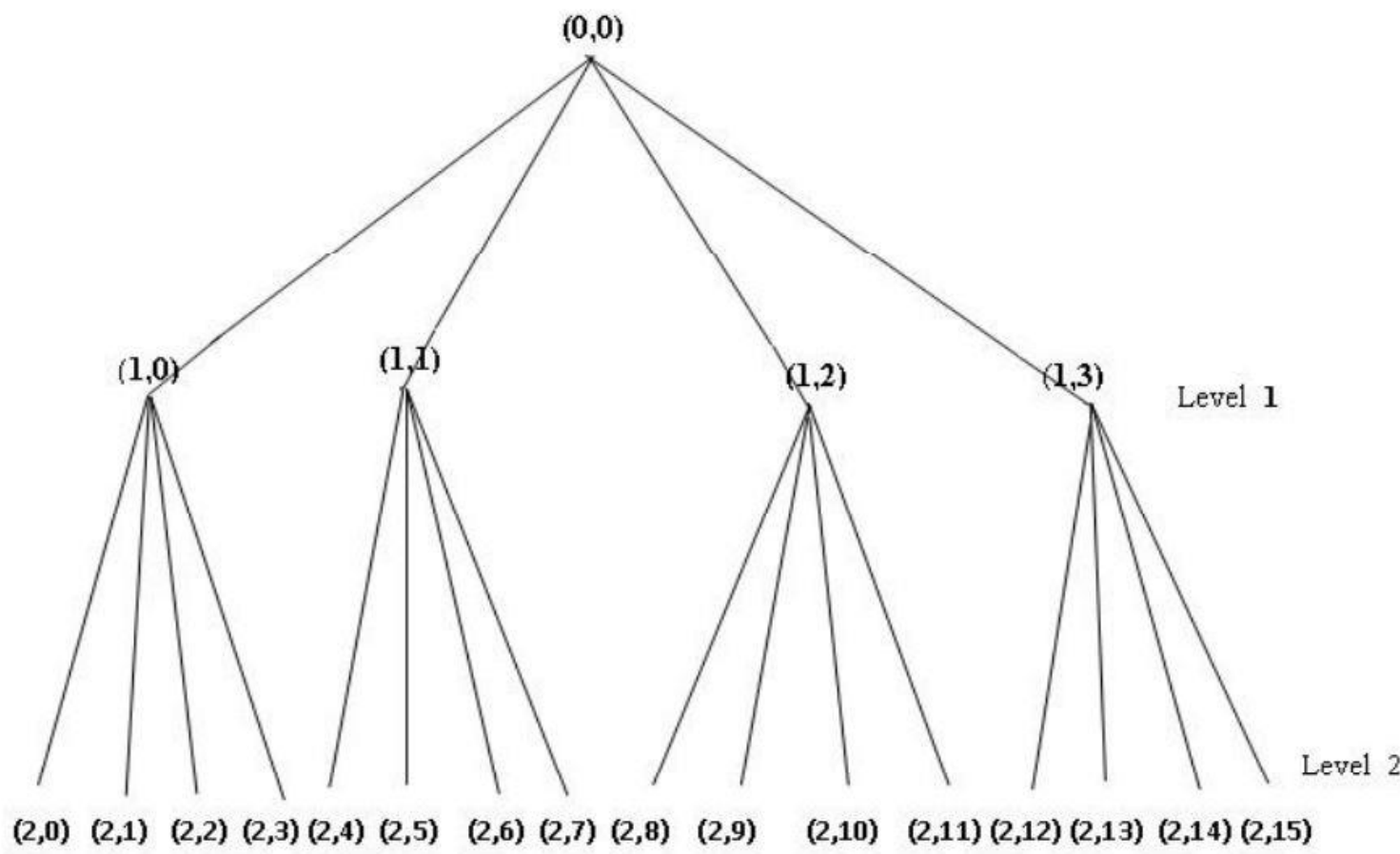

According to the traditional wavelet packet theory, the WPT of signal $\mathrm{x}(\mathrm{t})$ is defined as equation(1),

$$
x_{p}^{n . j}=2^{-j} / 2 \int_{R}^{-R} x(t) \mu_{n}\left(2^{-j} t-p\right) d t, 0 \leq j \leq s, 0 \leq n \leq 2^{s}-1
$$

Where $\mu_{\mathrm{n}}(\mathrm{t})$ is a wavelet packet transform, $\mathrm{j}$ is the number of decomposition level, $\mathrm{p}$ is the position parameter, $\mathrm{n}$ is the channel number, $\mathrm{s}$ is the maximum decomposition level. After decomposition by WPT, $2^{\mathrm{s}}$ sequence of the signal $x(t)$ can be obtained by the $S^{\text {th }}$ level as equations (2) and (3) shown below,

$$
\begin{aligned}
x_{k}^{2 n, j+1}=\sum_{p \in z} h(p-2 k) x_{p}^{n . j} \\
x_{k}^{2 n+1, j+1}=\sum_{p \in z} g(p-2 k) x_{p}^{n . j}
\end{aligned}
$$

\section{Micro Beam Structure}

Micro electro mechanical system (MEMS) technology dominate the present scientific research in almost all the domains including biological, medical and engineering field. MEMS is the present day technology which comprises with miniaturized electrical and mechanical parts for analysis. The input signal of the device may be force, pressure, heat or image. The MEMS device will actually transform the measured signal to a readable signal. There are four basic components of MEMS devices, micro sensor, micro actuators, micro structure and micro electronic device. 
Micro beams are considered to be an essential component for different sensing and actuation systems such as micro actuators, sensors, gyroscopes and resonators. Micro beams exactly anticipate the dynamic properties of the device, such as its natural frequencies and forced- vibration response. A micro beam which is one among the basic MEMS structure is very frequently used component for different sensing and actuation. Its simple geometrics and case of fabrication make them very appropriate for many applications. Measurement of stress is the mechanism which is linked with its sensitivity to some applied stimulation. As sensitivity is the deciding parameter of micro beams, any small defect in the structure, will affect the entire sensitive operation.

As the structure is miniaturized, the defect which occurs during fabrication or during operation may bring major impact in the output. So it is very necessary to fabricate micro beams without any defect and to monitor the structural health continuously for effective and maximum efficiency of the sensing unit. This study mainly deals with the identification or detecting the defects over the micro beams. Various methods were reported earlier for defect detection in macro scale using various technologies. But very few methods were adopted for micro devices. Here we impose the wavelet analysis to find the fault present in micro beams.

\subsection{Modeling of uniformly loaded Micro structured Beam}

In our study, we proposed the structure of micro beam using COMSOL multiphysics5.4 simulation software. Computer simulation is very essential in developing new products and for optimization of particular design. COMSOL is one which helps to develop and discover the accuracy of the model with real world precision. The model can be tested over various geometrical characteristic and physical parameters. Defining the geometries, material property and physics of the model can be encompassed with step by step modeling workflow. COMSOL multiphysics5.4 brings enhanced modeling features for modeling thin layered structure. Any combination of add on modules for various engineering applications can be simulated using this tool.

We had chosen poly crystalline silicon as device material due to its unique properties in fabricating micro structured devices[15]. Material properties of Poisson ratio - 0.22 , Density $-2320 \mathrm{~kg} / \mathrm{m}^{3}$ and young ${ }^{\mathrm{s}} \mathrm{s}$ modulus$160 \mathrm{Gpa}$ is chosen. Next the device parameters of length (L) $120 \mu \mathrm{m}$, width (W) $30 \mu \mathrm{m}$ and thickness (T) $1 \mu \mathrm{m}$ were fixed. Both the ends of the beam are fixed with the blocks of following parameters of length (L) $10 \mu \mathrm{m}$, width (W) $30 \mu \mathrm{m}$ and thickness (T) $5 \mu \mathrm{m}$. The force (W) applied is $3.75 \times 10^{-6} \mathrm{~N}$ and it is uniformly distributed over the beam.

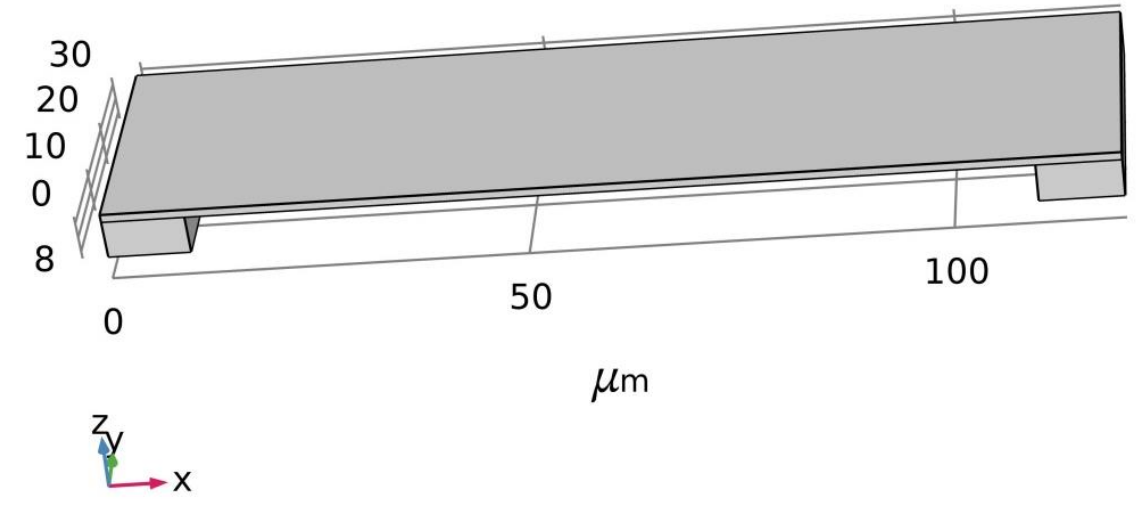

Figure. 3 Structure of Micro Beam fixed at both ends

For the above mentioned, material property and device dimensions, we calculate the deflection of the device theoretically using the equation 4 , since the beam will deflect in opposite direction of the force applied.

$$
\delta_{\max }=\frac{W L^{3}}{384 E I}
$$


Where, $\mathrm{E}$ is the young modulus of the material, I is the moment of inertia, $\mathrm{W}$ the force applied and $\mathrm{L}$ is the beam length, $\mathrm{W}$-width, T-thickness. Moment of inertia is given as equation 5 ,

$$
I=\frac{1}{12} W \cdot T^{3}
$$

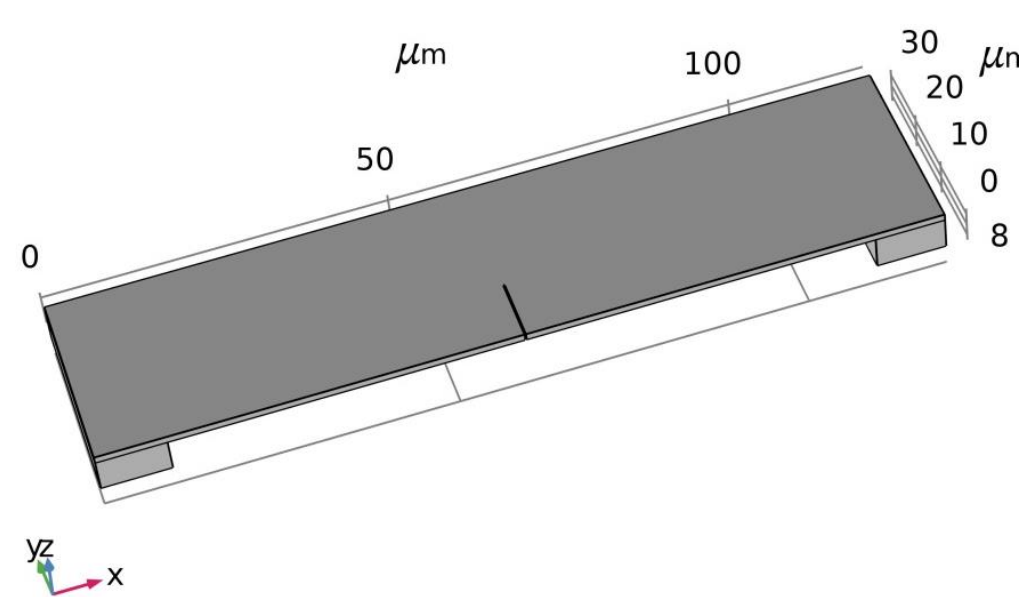

Figure.4 Structure of micro beam with crack in the middle

From the above equations, we calculated the theoretical value of maximum deflection to be $\delta_{\max }=0.0305 \mu \mathrm{m}$. For the purpose of locating cracks present in the device surface; we compared two devices with different crack which were intentionally made over the device structure. The constant crack dimensions of length $\mathrm{L}=0.2 \mu \mathrm{m}$, width $\mathrm{W}=10 \mu \mathrm{m}$ and thickness $\mathrm{T}=1 \mu \mathrm{m}$ were made at the length of $60 \mu \mathrm{m}$ which is exactly at the middle of the beam. Figure. 4 shows the schematic diagram of micro beam with crack in its surface.

\section{Results and Discussion}

\subsection{Deflection Analysis}

Whenever a force is applied to any structure or an object it will react with respect to the force applied. In the case of beams, when the force is applied the beams will deflect in the opposite direction of the force applied, however the applied force should be capable to make changes in the structure. When it is considered to be a micro structured beam, the force required to make the beam deflect also will be very minimum. Even a force of simple magnitude can make changes in the micro structure and the presence of defect or cracks in the beam structure can also produce greater impact in the device structure.
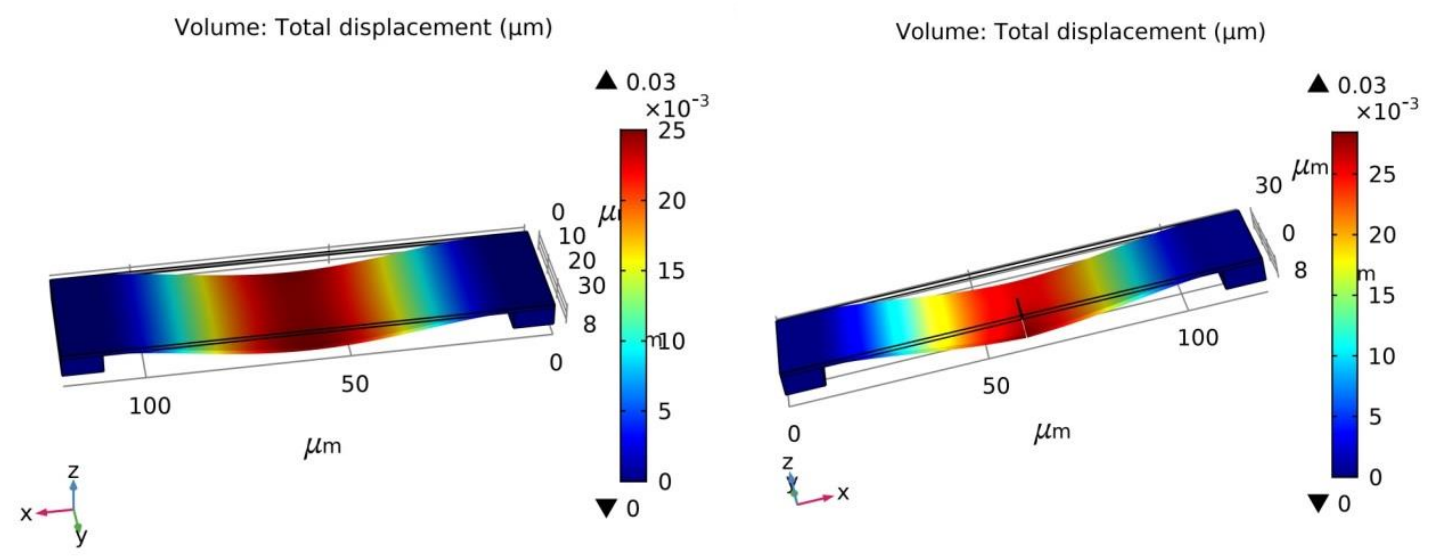

Figure.5 Displacement comparison of the beams with and without crack

Here for our study, the force of $3.75 \times 10^{-6} \mathrm{~N}$ is applied uniformly over the entire beam structure in $\mathrm{z}$ axis. As the result the beam will deflect in the opposite direction (y axis). The micro beam modeled in our study using COMSOL multiphysics, when subjected to the above said force, the beam deflect to the magnitude of $0.03 \mu \mathrm{m}$. 
this deflecting is almost equal and similar to our theoretically calculated deflection. Next for our study purpose, we introduce a crack exactly at the middle of the beam and executed the design for the same force. The beam with crack also shows the same deflection $0.03 \mu \mathrm{m}$ as of the beam without crack. So with the help of deflection analysis it is not possible to differentiate the beam structured with and without crack. Figure.5 shows the deformation of beams with and without crack for the force applied.

\subsection{Stress Analysis}

Stress is one among the important parameters which will be analyzed when monitoring the characteristics of a beam. As we discussed earlier, when a force is applied the beam will deflect with respect to the force applied. When the beam deformation occurs due to force applied, it will bend and elongate which build up stress in the beam structure. When the beam deflect the stress will be maximum at both fixed ends of the beam, then the stress will be gradually decreased. Here for the beam without crack we obtained a maximum stress of $5.83 \times 10^{6}\left(\mathrm{~N} / \mathrm{m}^{2}\right)$ near the fixed end. Then for the beam with crack at the center we observed the stress at the cracked region to be maximum next to the fixed ends. This is due to the structure defect, as the cracked region will be more exposed to the force applied. The beam with crack responds with the stress of $3.12 \times 10^{7}\left(\mathrm{~N} / \mathrm{m}^{2}\right)$ at the cracked position as represented by the Figure.6.
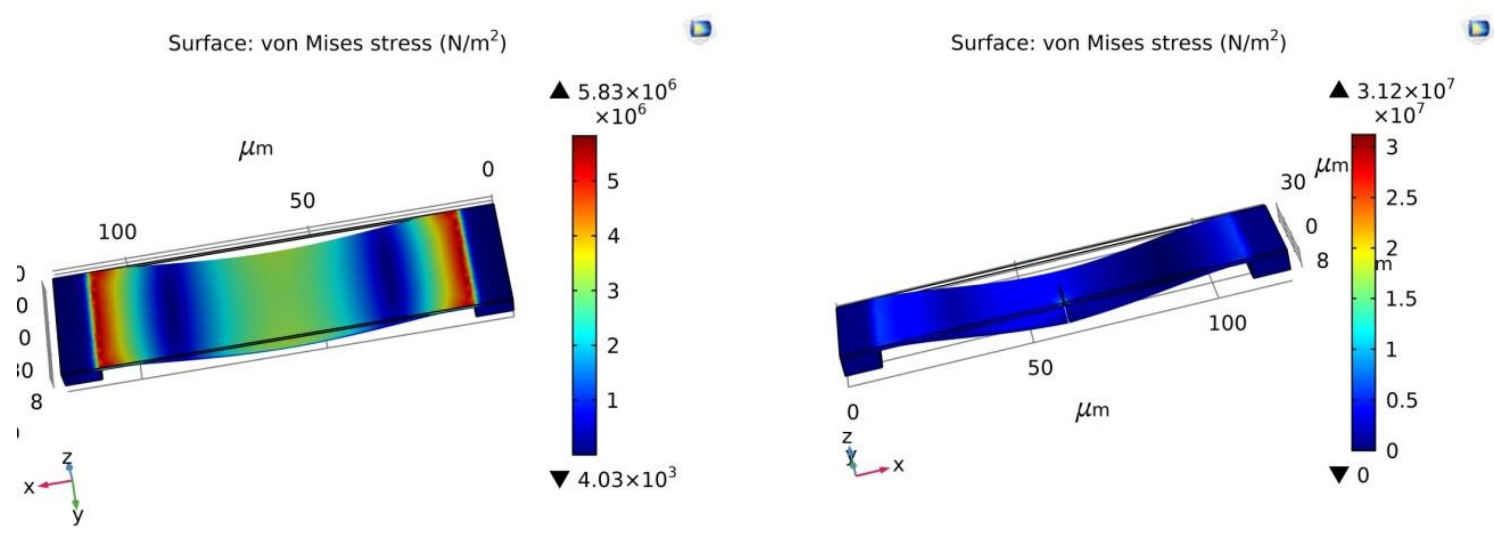

Figure.6 Stress Analysis of Beams

\subsection{Wavelet Analysis of the Beams}

The obtained stress values across the entire beam by the simulation were analyzed using Haar wavelet. The wavelet coefficient will responds directly proportional to the stress developed over the beam surface. Wavelet coefficient will results with ridges and peaks when the data discontinuity due to presence of crack or damages. Here in Figure. 7 we have shown the response of wavelet coefficients for Haar wavelet for the obtained results. The beam length of 120 is differentiated around 70 nodes and analyzed. For the healthy beam we can observe sharp peaks near the fixed ends of the beam where the stress will be maximum. In the beam with the crack, we obtained the resultant peaks around the region were the stress is maximum. In this case, we can clearly observe the peaks near the end and particularly a very sharp distractive peak at the exact location of the crack. By the use of graphs obtained, we can accurately interpret the crack location in the simple supported micro beams. 

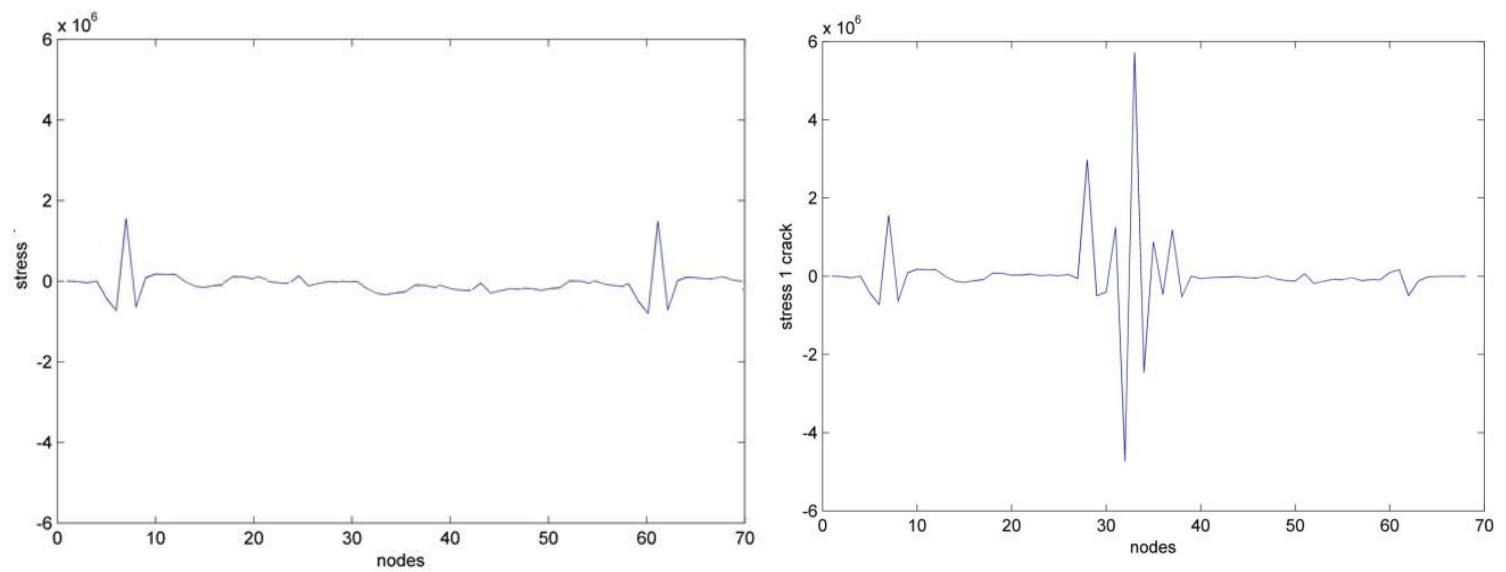

Figure.7 Stress Analysis of Beams using Haar wavelet

Figure. 8 and Figure. 9 implies the wavelet tree decomposition of the both healthy and cracked micro beam. The maximum level of wavelet decomposition node is chosen to be 6 . Every node in the wavelet tree is represented by original array and a range of elements.
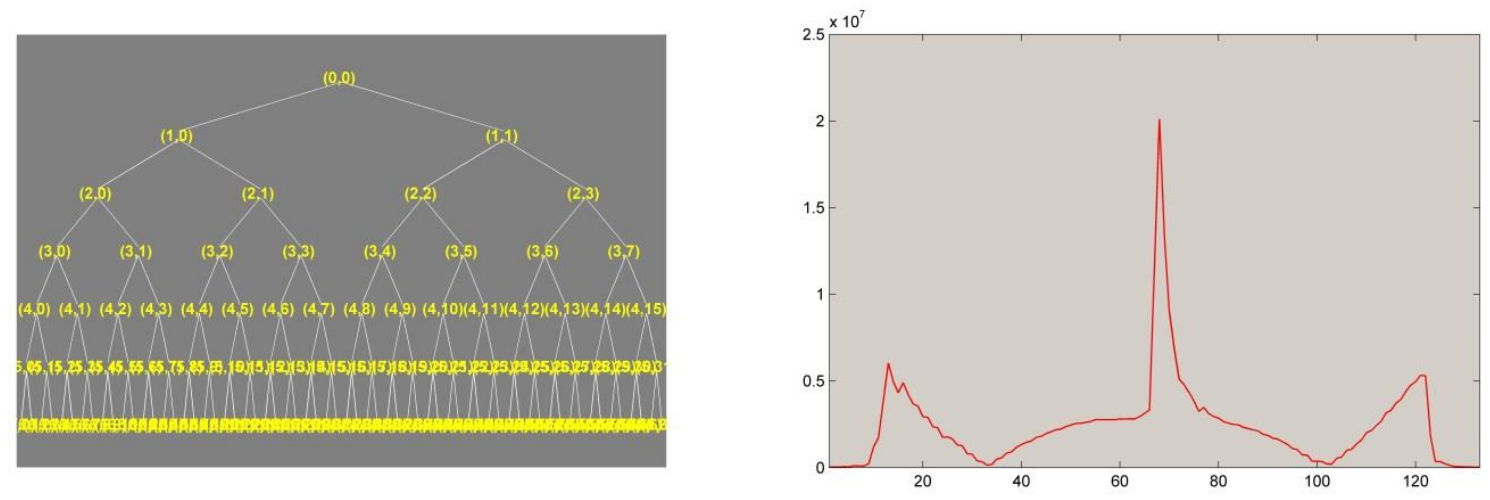

Figure.8 Wavelet Tree and Wavelet Packet Analysis of Healthy Beam
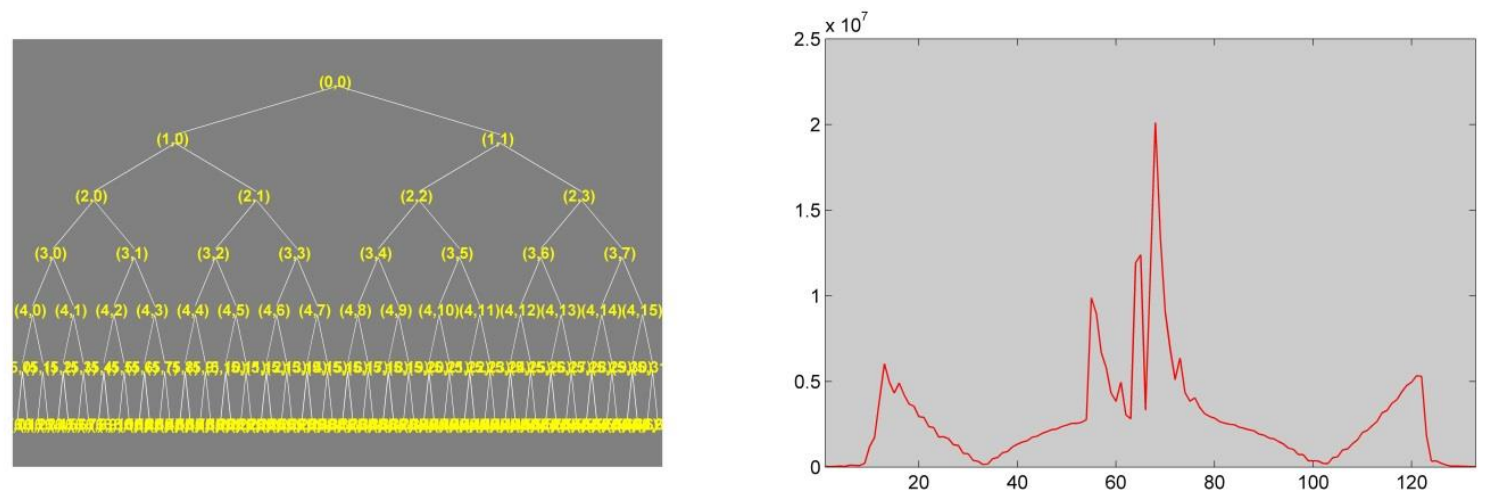

Figure.9 Wavelet Tree and Wavelet Packet Analysis of Cracked Beam

The peaks clearly represent the stress developed over the beam. In wavelet packet transform and signal decomposition, both the detail and the approximation coefficient are decomposed to produce full binary tree.

\section{Conclusion}

Our entire work, describes an efficacious method for locating the cracks present in the micro structure by wavelet transform analysis. Here we had chosen the micro beam which is supported at both ends. The required micro structure was modeled and simulated using COMSOL multi physics software. A uniform force is applied over the beam and the beam characteristics response of deflection and stress were obtained. The COMSOL results were compared for the healthy beam versus cracked beam and the finally analyzed using wavelet 
transform and wavelet packet transform. The graphs obtained by the both methods clearly describe the exact location of the crack. Since the micro structures should be fabricated and operated without any cracks, it is very essential to detect or locate the cracks in the micro devices. Our method can be efficiently used for such detective mechanism.

\section{References}

1. M. Sifuzzaman, M.R. Islam," Application of Wavelet Transform and its Advantages Compared to Fourier Transform" Journal of Physical Sciences, Vol. 13, 2009.

2. Lokenath Debnath," Wavelet Transforms and their Applications",PINSA-A,1998

3. Paul Embrechts, Agnes M. Herzbergb," An introduction to wavelets with applications to Andrews' plots" Journal of Computational and Applied Mathematics, 1995.

4. Daniel T.L. Lee, Akio Yamamoto, "Wavelet Analysis: theory and applications" Hewlett-Packard company,1994.

5. Jaber, Alaa \& Bicker, Robert. (2014). A Simulation of Non-stationary Signal Analysis Using Wavelet Transform Based on LabVIEW and Matlab. Proceedings - UKSim-AMSS 8th European Modelling Symposium on Computer Modelling and Simulation, EMS 2014. 138-144. 10.1109/EMS.2014.38.

6. Lee, Sang Kwon. (2002). A Acoustic Decay Measurement Based on Time-Frequency Analysis Using Wavelet Transform. Journal of Sound and Vibration - J SOUND VIB. 252. 141-153. 10.1006/jsvi.2001.4035.

7. Coifman, Ronald \& Meyer, Y. \& Quake, S. \& Wickerhauser, Mladen. (1993). Signal processing and compression with wavelet packets. Progress in Wavelet Analysis and Applications, Proceedings of the International Conference "Wavelets and Applications", Toulouse, 1992. 77-93.

8. Ezzodin, Amir \& Naderpour, H. \& Kheyroddin, Ali \& Amiri, Gholamreza. (2015). Damage Localization and Quantification of Beams Using Wavelet Transform. Journal of Modeling in Engineering. 12.

9. Ravikumar K,Ithaya Priya B. (2020) Identification of multiple crack locations in micro cantilever beam by wavelet transforms. Indian Journal of Science and Technology.13(35):36853694. https://doi.org/10.17485/IJST/v13i35.1472

10. Zhong, Shuncong \& Oyadiji, S.. (2007). Crack detection in simply supported beams without baseline modal parameters by stationary wavelet transform. Mechanical Systems and Signal Processing - MECH SYST SIGNAL PROCESS. 21. 1853-1884. 10.1016/j.ymssp.2006.07.007.

11. Lotfollahi-Yaghin, Mohammad \& Koohdaragh, Mahdi. (2011). Examining the Function of Wavelet Packet Transform (WPT) and Continues Wavelet Transform (CWT) in Recognizing the Crack Specification. Ksce Journal of Civil Engineering - KSCE J CIV ENG. 15. 497-506. 10.1007/s12205-011-0925-2.

12. Prieto, M. \& Novo, B. \& Manzanedo, F.. (2008). The Wavelet Packet Transform and its application to identify arc furnace current and voltage harmonics. 2008 IEEE Electrical Power and Energy Conference - Energy Innovation. 10.1109/EPC.2008.4763334.

13. brahim Turkoglu, Ahmet Arslan, Erdogan Ilkay, An intelligent system for diagnosis of the heart valve diseases with wavelet packet neural networks, Computers in Biology and Medicine, Volume 33, Issue 4,2003,Pages 319331,ISSN 0010-4825, https://doi.org/10.1016/S0010-4825(03)00002-7.

14. L. Eren and M. J. Devaney, "Bearing damage detection via wavelet packet decomposition of the stator current," in IEEE Transactions on Instrumentation and Measurement, vol. 53, no. 2, pp. 431-436, April 2004, doi: 10.1109/TIM.2004.823323.

15. Ertugrul, Ishak. (2020). micromachines The Fabrication of Micro Beam from Photopolymer by Digital Light Processing 3D Printing Technology. Micromachines. 11. 518. 10.3390/mi11050518. 